\title{
Acute Macroglossia Post Craniotomy in Sitting Position: A Case Report and Proposed Management Guideline
}

This article was published in the following Dove Press journal: International Medical Case Reports Journal

\author{
Omar Ababneh' \\ Subhi Alghanem' \\ Abdulrahman Al-Shudifat ${ }^{2}$ \\ Lubna Khreesha ${ }^{3}$ \\ Salameh Obeidat ${ }^{4}$ \\ Isam Bsisu (iD) \\ 'Department of Anesthesia and Intensive \\ Care, School of Medicine, The University \\ of Jordan, Amman, Jordan; ${ }^{2}$ Division of \\ Neurosurgery, Department of Special \\ Surgery, School of Medicine, The \\ University of Jordan, Amman, Jordan; \\ ${ }^{3}$ Division of Otolaryngology, Department \\ of Special Surgery, School of Medicine, \\ The University of Jordan, Amman, Jordan; \\ ${ }^{4}$ Department of Anesthesiology and \\ Perioperative Medicine, MD Anderson \\ Cancer Center, The University of Texas, \\ Houston, TX, USA
}

Background: Macroglossia is a rare life-threatening postoperative complication in patients undergoing neurosurgical operations in a sitting position. It is difficult to identify the cause of macroglossia, which can be considered multifactorial in most patients.

Case Presentation: We herein present a case of a 37-year-old female patient who was known to have a posterior occipital lesion (low-grade glioma with pilocytic features) and underwent occipital craniectomy followed by supratentorial approach for debulking of the tumor under general anesthesia in a sitting position. The patient developed upper airway edema along with extreme macroglossia immediately following extubation, with increasing difficulty in ventilation. Re-intubation was impossible, and urgent tracheostomy was performed. In the intensive care unit (ICU), the macroglossia worsened, and the patient developed sepsis with multi-organ failure and died 16 days postoperatively.

Conclusion: Acute macroglossia can be a catastrophic postoperative complication, necessitating early identification and a systematic approach to this critical event, in addition to being fully prepared to deal with difficult airway should this complication occur.

Keywords: macroglossia, postoperative complication, craniotomy, sitting position, airway obstruction, general anesthesia

\section{Introduction}

Acute tongue enlargement (Macroglossia) is a rare but potentially catastrophic postoperative complication following neurosurgical operations in a sitting position. ${ }^{1}$ Excessive cervical flexion along with local mechanical compression by any object in the pharynx such as endotracheal tube (ETT), throat pack, or transesophageal echocardiography probe (TEE) can impede or even obstruct the lymphatic and venous drainage, causing swelling of the upper airway, neck and face. ${ }^{2}$ In extreme and prolonged situations, arterial blood flow may also be compromised or even obstructed. Thus, after repositioning of the patient and relieving the mechanical pressure, ischemia reperfusion injury may occur, leading to severe upper airway edema and macroglossia within a few minutes. $^{2}$

In this case report, we present a 37-year-old female patient who underwent occipital craniectomy under general anesthesia in a sitting position, after which she developed severe macroglossia following extubation that required urgent tracheostomy.
Department of Anesthesia and Intensive Care, School of Medicine, The University of Jordan, PO Box 13046, Amman I 1942, Jordan

Tel +96265355000

Email omerababne@yahoo.com 


\section{Case Presentation}

A 37-year-old female patient known to have a posterior occipital lesion (low-grade glioma with pilocytic features) presented for occipital craniotomy followed by supratentorial approach for debulking of the tumor. The patient's physical status classification was II according to the American Society of Anesthesiologists Classification (ASA II), her physical exam where unremarkable, and she was not known to have any allergies.

The patient had previously undergone left parietal craniotomy and partial excision of the tumor in December, 1999 followed by chemotherapy and radiotherapy. Later, she started to have an increase in intracranial pressure (ICP) and underwent external ventricular drainage device (EVD) insertion under general anesthesia with no complications. In 2013, the patient was diagnosed to have recurrence of the brain tumor, and a right occipital craniotomy in a sitting position was done under general anesthesia that lasted for 8 hours in a sitting position without any postoperative complications.

In August 2014 she started to complain of blurred vision and was diagnosed to have a metastatic subthalamic brain tumor and was planned for a navigationassisted tumor debulking occipital craniotomy.

The surgical procedure lasted for around 10 hours and was performed in a sitting position with minimal head flexion and throat pack insertion. The procedure was invasively monitored, in which a left radial arterial line and right subclavian central line were inserted intraoperatively. Anesthesia was induced with propofol $(2 \mathrm{mg} / \mathrm{kg})$ and fentanyl $(1.5 \mu \mathrm{g} / \mathrm{kg})$. After muscle relaxation with cisatracurium $(0.15 \mathrm{mg} / \mathrm{kg})$ the airway was secured with a cuffed ETT with an internal diameter of $7.5 \mathrm{~mm}$, with Cormack-Lehane grade 1 view obtained by direct laryngoscopy, and the patient was connected to a mechanical ventilator. Anesthesia was maintained with isoflurane (1 MAC), cisatracurium (8 $\mathrm{mg} /$ hour) and remifentanil infusion. She was given intra-operatively 3.5 liters of Ringer lactate and one unit of packed red blood cells (PRBCs) and had an adequate urine output. Consequently, she had stable vital signs all through the procedure, systolic blood pressure around $100 \mathrm{mmHg}, \mathrm{PaCO}_{2}$ around 33 $\mathrm{mmHg}$ and oxygen saturation $99 \%$ all through the operation.

After the surgical intervention was finished, residual muscle paralysis was reversed with neostigmine $(0.05 \mathrm{mg} / \mathrm{kg})$ and atropine $(1.2 \mathrm{mg})$, a big throat pack was removed and after achieving spontaneous breathing in a supine position with a tidal volume (VT) of $800-850 \mathrm{~mL}$ and respiratory rate between 10 and 12 breaths/min with stable vital signs, extubation was done alongside. After extubation, a bedside neurological exam was done, which revealed left-sided weakness, and the neurosurgery team was informed.

While the neurosurgery was re-doing the slipped head dressing, we noticed a gradual decrease in the TV and a rapid increase in her tongue size, $\mathrm{O}_{2}$ saturation decreased to early $90 \mathrm{~s} \%$, and manual mask ventilation started with high resistance. Foreign body (throat pack, gauze) was thought to be the cause, so oropharynx was checked by laryngoscope, which showed enlarged tongue with an edematous soft palate. Moreover, epiglottis and vocal cords could not be seen (Cormack-Lehane grade 4), and the intubation was extremely difficult to be established. Mask ventilation became harder, the patient started to have inspiratory stridor and $\mathrm{O}_{2}$ saturation dropped transiently to the $80 \mathrm{~s} \%$. We called for help while $\mathrm{O}_{2}$ saturation was kept at 93-94\% by face mask manual ventilation. A fiber-optic trial was not done, because her airway needed to be secured in a time efficient fashion. The surgery team was consulted for urgent tracheostomy, while keeping bag-mask ventilation; though it was very hard to maintain manual ventilation due to increasing airway resistance. The tracheostomy was done without complications.

The patient was transferred to the intensive care unit (ICU) postoperatively, and kept on mechanical ventilation (Figure 1A), keeping her on sedation using propofol and remifentanil. She was also given antibiotics (cefuroxime, vancomycin, and levofloxacin), dexamethasone, and antihistamine regularly. Enteral feeding via a nasogastric tube was started and chest physiotherapy was done regularly.

Two days later (Figure 1B-D), she started to have more swollen tongue and neck. A mouth gag was applied, a magnetic resonance angiogram (MRA) was ordered, but unfortunately could not be done at that time. Five days later, she started to have an increase in white blood cells (WBCs) count and septic work-up was done, which revealed gram negative bacilli.

Nine days later (Figure 1E and F), her tongue began to increase in size again with ulcerations on the surface. Sixteen days later, she developed sepsis with multi-organ failure which ended with asystole and death was declared.

\section{Discussion}

Safe patient positioning involves balancing surgical comfort and optimal surgical setting against the risks related to 

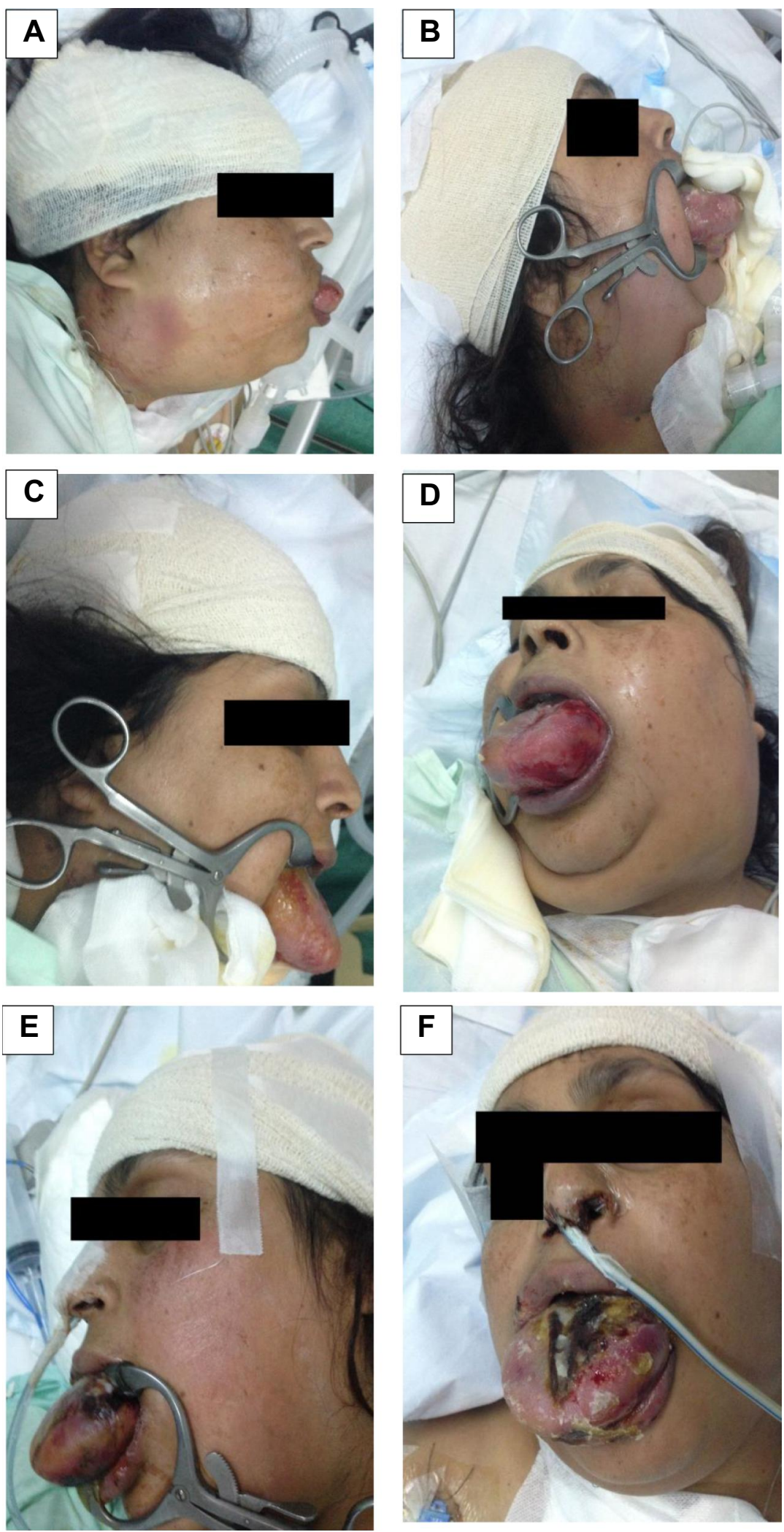

Figure I Postoperative macroglossia: (A) on the day of surgical intervention after being transferred to the ICU; (B-D) 2 days later, after which there was an increase in the swelling of the tongue and neck; (E-F): nine days later, after which she had a further increase in the size of the tongue with ulcerations on its surface. 
the patient position. ${ }^{3}$ Performing neurosurgical procedures in a sitting position can be challenging, requiring special attention from anesthesiologists. ${ }^{4}$

In the presented case, surgery and anesthesia lasted for 10 hours and were uneventful, with the incidence being noticed post-operatively. The estimated incidence of macroglossia in posterior fossa surgery in a sitting position is approximately $1 \%{ }^{5}$ However, the true incidence of macroglossia or acute tongue enlargement is unknown and there is a strong belief that it is underreported.

Although a sitting position provides better visualization of the surgical field and a clearer anatomical orientation, it is disfavored due to the risk of developing several intraoperative and postoperative complications. ${ }^{6}$ Complications associated with this position include: cervical spine strain, pressure necrosis of the chin, brachial plexus injury, as well as obstruction of cerebral venous and lymphatic outflow, which may lead to airway, neck, and face swelling, in addition to the development of macroglossia, which may be associated with airway obstruction. Moreover, an obstruction of the flow of cerebrospinal fluid, carotid artery, or vertebral arteries, paradoxical air embolism, and venous air embolism (VAE) may also develop, while pneumocephalus and subdural hematoma have also been reported. ${ }^{4,7,8}$ Likewise, bradycardia or cardiac arrest may also occur as a result of brainstem manipulations. ${ }^{4,7}$

Notably, it is difficult to identify the cause of acute macroglossia, which can be considered multifactorial in most cases. Causes can be classified into the following: 1) regional venous drainage obstruction due to extreme flexion of the neck and down position of the head, which can result in reperfusion injury-induced edema; 2) Mechanical compression of venous or lymphatic drainage of the tongue by ETT, laryngeal mask airway, bite block, TEE probe, other airway devices, throat packs, neurological monitoring and esophageal stethoscope. Other causes that may lead to macroglossia are allergic reaction, infection, hemorrhage, and neurogenic edema. ${ }^{9-11}$ Major risk factors for macroglossia are obesity, radiotherapy, chemotherapy, neck flexion, prolonged surgery, and multiple instrumentation. ${ }^{2,9}$

To date, no specific guideline for the management of acute postoperative macroglossia has been established. Remarkably, airway management can be challenging, and securing the airway in these patients is critical, which may require a surgical airway in many cases. ${ }^{12}$ Thus, several preventive measures for this serious complication should be addressed.
Preventive measures of acute macroglossia start from perioperative evaluation and identification of possible risk factors (Table 1). Intraoperatively, nasal intubation using cuffed flexometallic endotracheal tube is preferred to reduce the contact area and pressure at the base of the tongue. ${ }^{13,14}$ In addition, reducing surgical time as much as possible, avoiding extreme flexion, placing a bite block between the teeth, and avoiding oral instrumentation, throat packs and oral airways, in order to avoid overcrowding of the mouth and tongue depression. ${ }^{12,15,16}$ Proper positioning, intraoperative monitoring, and frequent assessment of the face and oral cavity intraoperatively can help in early detection. Moreover, simultaneous monitoring of central and jugular venous pressure can help in the assessment of hydration status and early detection of venous obstruction. ${ }^{15}$

Postoperatively, patients should be transferred to the ICU, where all oral instrumentations except endotracheal

Table I A Checklist of Perioperative Approach for Patients at Risk of Developing Postoperative Acute Macroglossia

\begin{tabular}{|l|}
\hline Preoperatively \\
\hline Identify possible risk factors: \\
- Previous history of head and neck radiotherapy. \\
- Previous history of chemotherapy treatment. \\
- Obesity. \\
Discussion of alternative surgical positions for the planned operation. \\
\hline Intraoperatively \\
Use of cuffed flexometallic endotracheal tube for nasal intubation (or \\
oral intubation in case nasal intubation was contraindicated or in case \\
of difficult nasotracheal intubation). \\
Avoid tongue compression injury: \\
- Place a soft bite block between teeth. \\
- Avoid oral instrumentation, throat packs, and oral airways. \\
- Avoid overcrowding of the mouth. \\
Proper head and neck positioning: \\
- Proper intraoperative patient positioning. \\
- Avoid extreme flexion. \\
- Check for signs of obstruction after positioning. \\
- Access to the patient's mouth and face should be guaranteed to \\
Proper intraoperative monitoring: \\
- Regular vital signs monitoring. \\
- Regular monitoring of pressure points. \\
- Rrequent assessment of the face and oral cavity. \\
Postoperatively \\
while still intubated. \\
- Regule monitoring of hydration status of the patient. \\
-
\end{tabular}




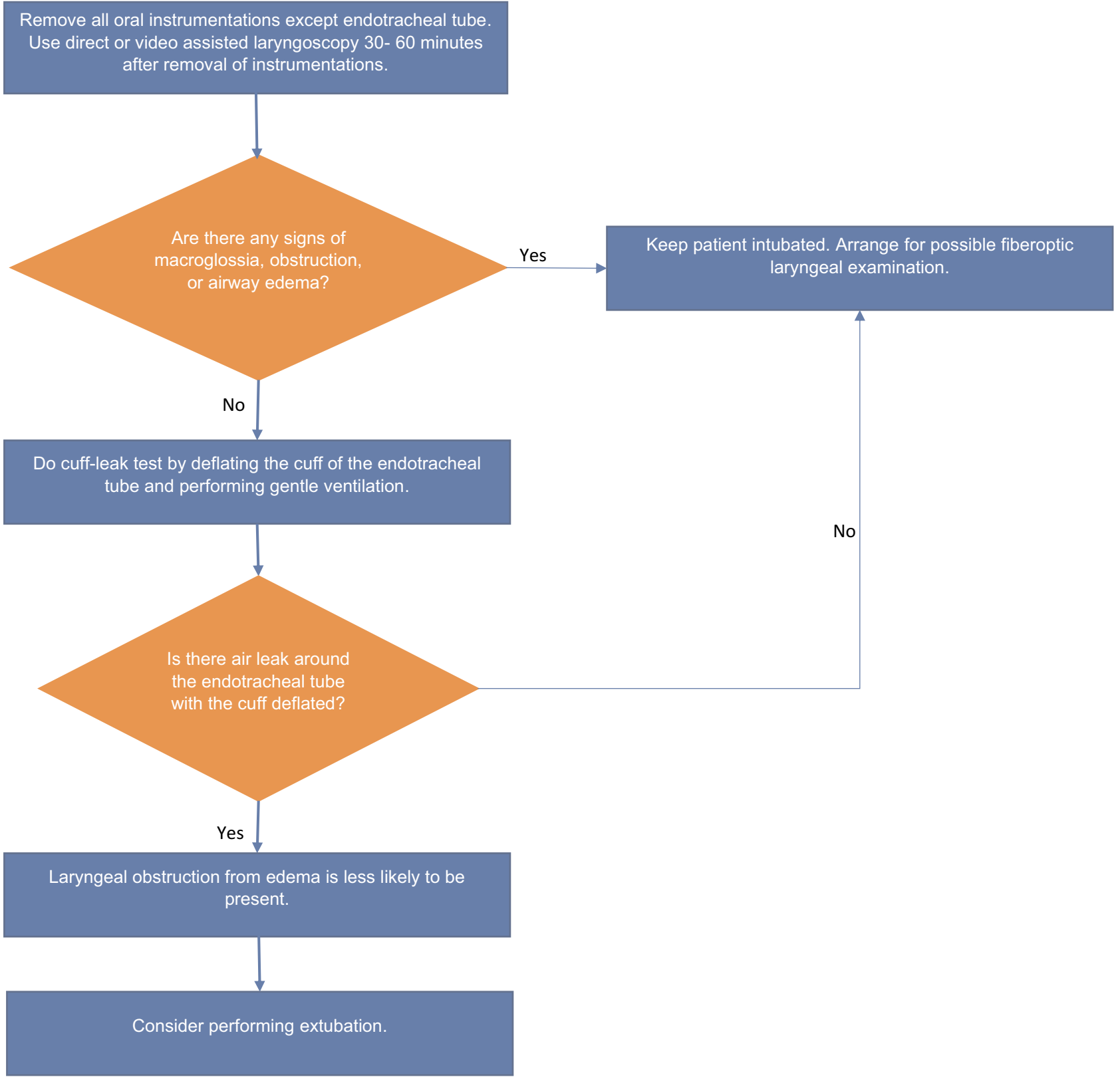

Figure 2 A flowchart of postoperative approach for considering extubation in patients at risk of developing postoperative acute macroglossia.

tube will be removed, and an airway assessment for signs of macroglossia or airway obstruction and edema will be done within 30-60 minutes via direct or video laryngoscopy. ${ }^{17}$ After direct airway assessment using laryngoscopy, a cuff leak test will be performed, which is a screening test for airway edema before extubation, in which the balloon cuff of the ETT is deflated and observation of the air passage around the ETT suggests that the airway is patent ${ }^{18}$ (Figure 2). After ruling out laryngeal obstruction, extubation may be considered. The anesthesia and intensive care teams must check and confirm the availability of all emergency re-intubation instruments prior to extubation. Post extubation, regular vital signs monitoring and frequent physical examination of the face and airway must be performed, in order to ensure early identification of airway edema and postextubation complications ${ }^{19}$ (Figure 3).

In conclusion, acute macroglossia can be a catastrophic postoperative complication following surgeries done in a sitting position. This necessitates early identification of 
Ensure that all instruments needed for emergency re-

intubation are available, and that tracheostomy team is

available in case of failed emergency intubation.



Figure 3 A flowchart of post-extubation follow-up and monitoring in patients at risk of developing postoperative acute macroglossia.

this critical complication in addition to being fully prepared to deal with difficult airway should this complication occur.

\section{Consent for Publication}

A written informed consent was obtained from the patient's family regarding the publication of this case and accompanying images. The ethics committee at our institution does not usually require the approval of the Institutional Review Board (IRB) for case reports.

\section{Author Contributions}

All authors made a significant contribution to the work reported, whether that was in the conception, study design, execution, acquisition of data, analysis and interpretation, or in all these areas; they took part in drafting, revising or critically reviewing the article; gave final approval of the version to be published; have agreed on the journal to which the article has been submitted; and agree to be accountable for all aspects of the work.

\section{Funding}

This research received no funding.

\section{Disclosure}

The authors report no conflicts of interest in this work.

\section{References}

1. Figueredo-Gaspari E, Fredes-Kubrak R, Canosa-Ruiz L. [Macroglossia after surgery of the posterior fossa]. Rev Esp Anestesiol Reanim. 1997;44(4):157-158. Spanish.

2. Lam AM, Vavilala MS. Macroglossia: compartment syndrome of the tongue? Anesthesiology. 2000;92(6):1832-1835. doi:10.1097/00000 542-200006000-00046

3. Rozet I, Vavilala MS. Risks and benefits of patient positioning during neurosurgical care. Anesthesiol Clin. 2007;25(3):631-x. doi:10.1016/j. anclin.2007.05.009

4. Schramm P, Tzanova I, Goock T, et al. Noninvasive hemodynamic measurements during neurosurgical procedures in sitting position. J Neurosurg Anesthesiol. 2017;29(3):251-257. doi:10.1097/ANA.00 00000000000300

5. Moore JK, Chaudhri S, Moore AP, Easton J. Macroglossia and posterior fossa disease. Anaesthesia. 1988;43(5):382-385. doi:10.1111/ j.1365-2044.1988.tb09018.x

6. Gupta P, Rath GP, Prabhakar H, Bithal PK. Complications related to sitting position during pediatric neurosurgery: an institutional experience and review of literature. Neurol India. 2018;66(1):217-222. doi: $10.4103 / 0028-3886.222852$

7. Srivastava S. The sitting position for neurosurgery: a bane or a boon. Neurol India. 2018;66(1):223-225. doi:10.4103/0028-3886.222830

8. Malhotra S. Venous air embolism in neurosurgical patients. In: Khan Z, editor. Challenging Topics in Neuroanesthesia and Neurocritical Care. Springer, Cham; 2017:229-238. 
9. Sharma PK, Bhakta P, Srinivasan S, Khan RM, Kaul N. Acute tongue enlargement secondary to pharyngeal packing after tracheal intubation-a case report. Middle East J Anaesthesiol. 2012;21(5):761-764.

10. Toyama S, Hoya K, Matsuoka K, Numai T, Shimoyama M. Massive macroglossia developing fast and immediately after endotracheal extubation. Acta Anaesthesiol Scand. 2012;56(2):256-259. doi:10. 1111/j.1399-6576.2011.02575.x

11. Vermeersch G, Menovsky T, De Ridder D, De Bodt M, Saldien V, Van de Heyning P. Life-threatening macroglossia after posterior fossa surgery: a surgical positioning problem? B-Ent. 2014;10(4):309-313.

12. Brockerville M, Venkatraghavan L, Manninen P. Macroglossia in neurosurgery. J Neuroanaesthesiol Crit Care. 2017;4(02):078-84. doi:10.4103/jnacc-jnacc-64.16

13. Morrison G, Tobin JM. Endotracheal intubation: oral and nasal. In: Demetriades D, Inaba K, Lumb P, editors. Atlas of Critical Care Procedures. Springer, Cham; 2018:3-14.

14. Perry JL. A Comparison of Gas Flow Resistance in Parker Flex-Tip and Mallinckrodt RAE Nasal Endotracheal Tubes. The Ohio State University; 2013.
15. Ha JF, Vitale LN, Pfarr MA, Kawai Y, Zopf DA. Massive macroglossia after posterior cranial fossa surgery: a case report. A a Pract. 2018;10(8):204-208. doi:10.1213/XAA.0000000000000663

16. Bouaoud J, Joly A, Picard A, et al. Severe macroglossia after posterior fossa and craniofacial surgery in children. Int J Oral Maxillofac Surg. 2018;47(4):428-436. doi:10.1016/j.ijom.2017.12.003

17. Bhatnagar V, Jinjil K, Dwivedi D. Time to include video laryngoscope as a tool for extubation in difficult airway cases! Indian J Anaesth. 2019;63(8):677-678. doi:10.4103/ija.IJA_249_19

18. Lewis K, Culgin S, Jaeschke R, et al. Cuff leak test and airway obstruction in mechanically ventilated ICU patients (COMIC): a pilot randomised controlled trial protocol. BMJ Open. 2019;9(7): e029394. doi:10.1136/bmjopen-2019-029394

19. Pluijms WA, van Mook WN, Wittekamp BH, Bergmans DC. Postextubation laryngeal edema and stridor resulting in respiratory failure in critically ill adult patients: updated review. Crit Care. 2015;19(1):295. doi:10.1186/s13054-015-1018-2

\section{Publish your work in this journal}

The International Medical Case Reports Journal is an international, peer-reviewed open-access journal publishing original case reports from all medical specialties. Previously unpublished medical posters are also accepted relating to any area of clinical or preclinical science. Submissions should not normally exceed 2,000 words or 4 published pages including figures, diagrams and references. The manuscript management system is completely online and includes a very quick and fair peer-review system, which is all easy to use. Visit http://www.dovepress.com/testimonials.php to read real quotes from published authors. 